\title{
5 Three billboards to support ethical-legal adolescent HIV prevention research in Eastern and Southern Africa
}

\author{
Ann E. Strode and Catherine M. Slack
}

\section{Introduction}

It is a universally accepted legal norm that children (individuals under the age of 18) have fundamental human rights, like adults, and these rights may only be limited in justifiable circumstances (United Nations General Assembly: Convention on the Rights of the Child [CRC], 1989; Teddy Bear Clinic for Abused Children and Another v Minister of Justice and Constitutional Development, 2013). The South African Constitutional Court has succinctly set out the rationale for this approach by stating:

Every child has his or her own dignity. If a child is to be constitutionally imagined as an individual with a distinctive personality, and not merely as a miniature adult waiting to reach full size, he or she cannot be treated as a mere extension of his or her parents.

$(S \approx M, 2007$, p. 11)

The Convention on the Rights of the Child (CRC) is premised on the principle that all children are right bearers with inherent dignity (United Nations General Assembly, 1989). It is underscored by four principles, namely the right of every child to not be discriminated against; to survival and development; to have their best interests accounted for; and to be involved in decision-making which impacts them (United Nations General Assembly, 1989). These principles ought to inform the way in which we approach all public health programming for children, including the development of new HIV prevention interventions.

There is a critical need to evaluate whether HIV prevention modalities are safe and effective for use in a sub-set of children, namely, adolescents (Abdool-Karim and Dellar, 2014). Adolescents are often defined as persons aged 10-19 and this broad definition is widely accepted, even though it does not fit neatly with legal definitions that end childhood at 18 (United Nations General Assembly, 1989). In this chapter, we will highlight the complexities of undertaking HIV prevention research with persons who have evolving capacity to make health-related choices and are accorded special protections in law. 
Prior work has examined how the principles in the CRC can be utilized to consider thoughtful involvement of adolescents in HIV prevention studies (Busza et al., 2016). This chapter builds on that work by critically discussing the legal and ethical complexities of involving adolescents in HIV prevention studies in Eastern and Southern Africa (ESA).

Inspired by the title of the 2017 Hollywood film (Three Billboards outside Ebbing, Missouri), this chapter first highlights that including adolescents in HIV prevention studies is legally and ethically justified; second, it emphasizes that the legal ethical complexities of involving them are resolvable; and third, it outlines how various stakeholders can "come to the table" to promote adolescent enrolment in HIV prevention trials.

\section{Billboard 1 - adolescent HIV prevention research is legally and ethically justified}

A fundamental human right is, as the International Covenant on Economic, Social and Cultural Rights (ICESCR) records: everyone is entitled to "the highest attainable standard of physical and mental health" (United Nations Office of the High Commissioner for Human Rights [OHCHR], 1966, p. 4). The CRC uses the same terminology when describing children's rights (United Nations General Assembly, 1989). The African Charter on Human and People's Rights (ACGPR) formulates this in a similar manner, stating that all people have the right to the "best attainable state of physical and mental health" (African Commission on Human and Peoples' Rights [ACHPR], 1981, p. 3). It is argued that to achieve this goal (of "best" or "highest" "attainable standard" of healthcare), ongoing research is required to develop and improve interventions, treatments and services.

Furthermore, when research is undertaken, everyone has the right to benefit from it. Article 15 of the ICESCR states that everyone is entitled to "enjoy the benefits of scientific progress and its applications" (OHCHR, 1966 , p. 5). Recently, this has been referred to as a "right to science" (Scanlon et al., 2017). It is suggested that the right has three core elements, all of which place obligations on the state (Scanlon et al., 2017). These elements are, first, an obligation on the state to ensure everyone benefits from science; second, a right to contribute to scientific advances and a freedom to undertake research; and third, a right to be involved in scientific decisionmaking (Scanlon et al., 2017).

The CRC does not itself expressly refer to a child's rights regarding research or science. However, point 29 of the General Comment No. 3 (2003) on HIV/AIDS and the Rights of the Child issued by the Committee on the Rights of the Child emphasizes:

Consistent with article 24 of the Convention, State parties must ensure that HIV/AIDS research programmes include specific studies that 
contribute to effective prevention, care, treatment and impact reduction for children.

General Comment No. 5 of 2003 asserts further that states should ensure that this is done in a particular way:

The Committee emphasizes that, in many cases, only children themselves are in a position to indicate whether their rights are being fully recognized and realized. Interviewing children and using children as researchers (with appropriate safeguards) is likely to be an important way of finding out, for example, to what extent their civil rights, including the crucial right set out in article 12, to have their views heard and given due consideration, are respected within the family, in schools and so on.

(UN Committee on the Rights of the Child [CRC], 2003, p. 41)

The approach in this General Comment reflects a shift towards protecting children from unsafe, ineffective interventions through data obtained from children themselves, via rigorous studies, and away from protecting children from research participation per se (Nelson et al., 2010). The General Comments quoted above show that the Committee on the Rights of the Child (the body which ensures implementation of the CRC) interprets the health rights within the CRC to include child participation in research.

Likewise, there are ethical arguments for including adolescents in HIV prevention trials that can be made through the application of key ethical principles - beneficence and justice, based on various international ethical guidelines.

The principle of beneficence motivates the inclusion of children in research because it requires researchers to strive for the greater good. In the case of HIV prevention research, this point has been argued because HIV is a significant health threat and health challenge for many adolescents, including in Africa:

Beneficence provides an ethical basis for conducting research that may improve health and a basis for maximizing the benefit of research and minimizing its risk. Research with adolescents may have important benefits to individual adolescents, and it may benefit adolescents as a group as well.

(Santelli et al., 2003, p. 398)

Accordingly, the ethical principle of beneficence as grounds to promote the welfare of enrolled children, and to consider benefits to "children as a social group ... through the implementation of evidence-based 
policy-practice" is a recommended consideration for researchers (UNICEF, 2013, p. 17).

The ethical principle of non-maleficence (to avoid harm) can also be invoked to enable inclusion of children/adolescents in research. For example, not testing products on adolescents can result in their exposure to interventions that are potentially ineffective and possibly harmful:

In general, this lack of information results in higher risks for . . . adolescents from being exposed to interventions where little is known about their specific effects or safety in this population. Therefore, it is imperative to involve . . . adolescents in research.

(Council for International Organizations of Medical Sciences [CIOMS], 2016, p. 66)

Also, the ethical principle of justice underpins efforts to include adolescents in research, as it requires adolescents to be treated equally:

The principle of justice demands a fair sharing of both risks and benefits. If certain groups of persons are systematically excluded from participation in research, these groups may not share in the beneficial results of that research. Promoting full participation by groups that historically have been excluded from research and its benefits is founded on the principle of justice. The interests of justice demand that adolescents ... not be excluded from participation in research that may have direct or indirect benefit.

(Santelli et al., 2003, p. 398)

Another ethical principle that is relevant is that of respect for persons (Santelli et al., 2003), which encourages researchers to show respect for the evolving ability of adolescents to make decisions about participation in research and to recognize adolescent limitations in judgement when consent approaches are designed.

When international ethical-legal principles are applied in the context of HIV prevention, it is argued that enrolling adolescents in such research is justified both legally and ethically. First, using the Scanlan, Macnaughton and Sprague approach it is submitted that countries have a responsibility to ensure that the highest attainable standard of healthcare is provided to children. As stated previously, adolescents are at an increased risk of contracting HIV and interventions are necessary to address their particular vulnerability to infection (Bekker et al., 2015; Hosek and Zimet, 2010; Shah et al., 2018; Osmanov, 2007). More specifically, adolescent behaviours place them at risk of HIV (e.g. early sexual debut, inconsistent condom use). Features of adolescence may also heighten their HIV risk (e.g. sensation-seeking). Structural factors may impact their risk (e.g. inadequate frameworks for 
their access to services). Adolescents constitute a key population for HIV intervention including for biomedical approaches (Hosek and Zimet, 2010; Wilson et al., 2010).

Extrapolation of data from adult studies (e.g. on safety, acceptability, efficacy) may be very difficult. Even where such extrapolations may be made, some studies will be necessary to answer specific questions of safety, feasibility, acceptability or adherence in the adolescent group given the differences in the way adolescence may react to, metabolize or respond to interventions only tested in adults. Also, regulatory approval will likely require data from this group (for "youth labelling indications," i.e., detail in the package insert which describes dosage and possible side effects for young people). Ideally this should be concurrent with adult product licensure to prevent off-label use (Kapogiannis et al., 2010), where adolescents make use of prevention products in an manner unsupported by evidence or regulatory approval.

In summary, it is argued that failing to address the needs of adolescents for HIV prevention violates their right to the highest attainable standard of healthcare as provided for in both the CRC and the ICESCR. This argument is supported by the wording in Article 24(2)(f) of the CRC that requires state parties to "develop preventive health care" as part of a child's right to health (CRC, 2003, p. 172). Arguably, preventive healthcare cannot be developed without research involving adolescents. This interpretation of Article 24 in the CRC is confirmed by General Comments 3 and 5, referred to earlier, both of which require research to ensure the promotion of children's right to health.

The legal rights-based argument - that research is an essential element of the right to ensure the highest attainable standard of health - is supported by the approach in ethics guidelines in many countries. South African ethics guidelines remind researchers that while adolescents should not bear research burdens unnecessarily, adolescents "are entitled to improved health care based on findings drawn from rigorous research conducted in the child population" (South Africa Department of Health [SA DOH], 2015, p. 28). Researchers are also encouraged to avoid the systematic exclusion of "vulnerable participants because to do so is unfairly discriminatory and vulnerable persons are potential beneficiaries of relevant research" (SA DOH, 2015, p. 8). This ethical approach is similar to the legal one, as it is premised on obligations to achieve a high standard of health.

Second, failing to undertake adolescent HIV prevention studies, and excluding adolescents as participants, violates adolescents' rights to equality. It would be wrong for adults to "enjoy the benefits of scientific progress" while adolescents do not, insofar as they access interventions with little safety or effectiveness data for their age category (OHCHR, 1966, p. 5; Strode et al., 2014).

Third, using the Scanlan et al. approach, it is submitted that a core part of the right to science is to participate in its development (Scanlon et al., 2017). Excluding adolescent participants from studies of HIV prevention 
means that we violate one of the one core principles underpinning the CRC, that of child participation. This impacts directly on the interventions we are trying to develop for this age category. Ivan-Smith and Johnson (1998) have stated that vital information is needed in order to develop solutions that will address adolescent concerns.

It also undermines adolescents' right to dignity $(S v M, 2007)$. The CRC recognizes that every child has inherent worth (United Nations General Assembly, 1989). Delaying adolescents access to potentially life-saving interventions to prevent HIV violates their dignity as it shows a lack of commitment to addressing their particular needs, implying they are of less value in a national HIV prevention programme.

Likewise, ethics guidance suggests a need to recognize the "positive benefits that well-conducted clinical research can bring, and the dangers of providing healthcare that is not underpinned by a solid evidence base" (Nuffield Council on Bioethics, 2015, p. 80).

\section{Billboard 2 - stakeholders must prepare for complexities related to adolescent capacity and adolescent behaviour}

It is evident that adolescent enrolment in HIV prevention studies is critical, yet features of adolescence may potentially heighten risk of study-related harm and potentially undermine comprehension required for agreement to take part. For example, sensitivity to peer evaluation may heighten their experiences of stigma from trial participation, or deficiencies in abstract reasoning or conceptual thinking and experiential immaturity may compromise their ability to understand research and research-related risks (MacQueen and Abdool-Karim, 2007). Furthermore, HIV prevention studies may involve invasive procedures with the potential for risk and burden. Adolescents may undergo sexual risk assessments, contraceptive compliance assessments, STI and/or HIV testing and product administration (Bekker, 2014, 2015).

In ESA, the enrolment of adolescents in HIV prevention studies is not a simple endeavour. Complexities relate to regulatory frameworks that do not adequately address issues relating to the study population (adolescents). Some frameworks also provide insufficient guidance on complexities related to the study questions and procedures (e.g. sensitive, stigmatized behaviours related to sexual activity or identity, such as same-sex behaviour). The following sections describe some of these complexities and ways in which researchers, research ethics committees and other stakeholders can respond to them.

\section{Adolescent capacity}

The complexity with involving persons under the age of 18 in studies is that they lack full legal capacity (United Nations General Assembly, 1989; World Health Organization [WHO], 2018). This generally necessitates 
the assistance of an adult entrusted with decision-making authority, which is usually the parent or guardian (Strode et al., 2010; WHO, 2018). It is generally recognized that parents/guardians generally are the individuals who would have knowledge of what their child needs are, would protect them from risks and hazards and would protect their interests (Santelli et al., 2017), given adolescents' inexperience regarding decision-making in real-world situations (Santelli et al., 2003). This has led to many countries requiring parental/guardianship permission for adolescent participation in studies (CIOMS, 2016).

Responses to lack of adolescent capacity are reflected in the ethical-legal frameworks of several ESA countries, where adolescents are only able to participate in health research with parental or guardianship consent (Grant and Patel, 2016). Table 5.1 provides information from four countries to illustrate this point.

Table 5.1 shows that in some ESA countries hosting prevention studies, the legal framework narrowly confines acceptable consent strategies to parental/guardianship consent. This consent strategy means dual decisionmaking (parental permission and adolescent assent), and it is not straightforward because:

1 Some adolescents may not be cared for on a day-to-day basis by their parents or legal guardians (Strode and Slack, 2011).

2 The values and preferences of both parties may not be aligned.

3 There may be power disparities between children and adults, and some societies may place a high social value on obedience, which might tempt adolescents to align their decisions to parental preferences over their own.

4 Most critically, many adolescents generally do not want their parents to know about sexual activity or sexual orientation that might attract parental disapproval or sanction (Abdool-Karim and Dellar, 2014; Gilbert et al., 2015; Hosek and Zimet, 2010; Knopf et al., 2017; Shah et al., 2018; Wallace et al., 2018).

RECs reviewing trials in such settings may be sympathetic to the above problems; nevertheless, they face an ethical-legal dilemma in that they are required to act ethically and to comply with legal obligations. Where there

Table 5.1 The legal age of consent to health research in selected ESA countries

\begin{tabular}{ll}
\hline Country & Age of independent consent to health research \\
\hline Ethiopia & 18 \\
Kenya & 18 \\
Mozambique & 21 \\
South Africa & 18 \\
\hline
\end{tabular}


is a conflict between law and ethics the REC ought to seek advice on the way forward. A principled position should be developed and institutional support sought to ensure consistency in REC decision-making.

The situation is also complex because although children do not have full legal capacity until they are 18 , their capacity is constantly developing. The CRC recognizes that (a) as adolescents get older, their capacity evolves; and (b) even where adolescents lack full legal capacity, they ought to be involved in decisions that affect them (United Nations General Assembly, 1989; WHO, 2018).

Some countries have acknowledged this and passed legislation that recognizes the capacity of children under the age of 18 to consent independently to various sexual and reproductive health interventions (Shah et al., 2018). However, other countries, particularly in ESA, have been slow to pass laws that provide adolescents the right to consent independently to sexual and reproductive interventions (see Table 5.2). Where the law is silent on this issue, it is presumed that adolescents in such settings will require parental or guardianship consent for the listed interventions.

Reading Tables 5.1 and 5.2 together, this means that in some ESA countries hosting prevention studies, parents are required to give permission for both enrolment and various study-related components. In such instances, adolescent participants will have little expectation of privacy for components where parents have given permission. However, study participation itself may be even less appealing for adolescents, who may not wish their parents to consent for and receive information about sensitive components such as STI or HIV testing or contraception use (Hosek and Zimet, 2010; Strode and Essack, 2017); this may deter them from enrolment.

Table 5.2 Ages of consent in selected ESA countries to medical treatment, contraceptives and HIV testing

\begin{tabular}{llll}
\hline Country & Medical treatment & Contraceptives & HIV testing \\
\hline Botswana & 16 & 16 & 16 \\
DRC & Unclear & Unclear & 18 \\
Ethiopia & 18 & 18 & 15 (policy) \\
Kenya & Unclear & Unclear & 18 \\
Lesotho & 12 & 12 & 12 \\
Malawi & - & - & 13 \\
Mozambique & - & - & 16 \\
Namibia & 14 & - & 14 \\
Rwanda & - & 15 & 15 \\
South Africa & 12 & 12 & 12 \\
Eswatini & 12 & 12 & 12 \\
Tanzania & - & - & 18 \\
Uganda & - & - & 12 \\
Zambia & - & - & 16 \\
Zimbabwe & - & - & 16 \\
\hline
\end{tabular}

Source: Grant and Patel (2016). 
Again reading Tables 5.1 and 5.2 together, this also means that in some ESA countries, parents are required to give permission for enrolment into HIV prevention studies but are not required to give permission for various components adolescents will likely receive in such studies. For example, in three countries adolescents require parental consent for study enrolment below age 18 but can self-consent to HIV testing below age 18 . These are Ethiopia (HIV testing 15, research 18), Mozambique (HIV testing 16, research 21) and South Africa (HIV testing 12, research 18). In such instances, careful thought must be given as to how researchers will respect adolescents' rights to consent to certain sexual and reproductive components within the context of research where parental consent has been sought for enrolment.

\section{Adolescent behaviour}

Another category of ethical-legal complexities relates to the adolescent behaviours that may be uncovered or explicitly researched during studies of HIV prevention. These complexities often flow from an intersection between the research questions and procedures on the one hand and the criminal law on the other hand. Some HIV prevention studies might enrol at-risk or high-risk adolescents to answer key questions about the safety, tolerability or efficacy of products for HIV prevention. In such studies, sexual activity may be an eligibility criterion for enrolment. Even if this is not the case, the research team may come to know (in some way or another) about adolescent sexual activity (e.g. through behavioural questionnaires, in-depth interviews, tests or examinations, or even inadvertent disclosure) (Strode and Slack, 2009). In many countries, persons cannot lawfully consent to sex below the age of 18, for example in Ethiopia, Kenya, Mozambique, Rwanda, Tanzania and Uganda (see Table 5.3). In such instances, researchers should establish if there are mandatory reporting obligations on researchers to report adolescent participants who are breaking the law by engaging in underage but consensual sexual activity. Here researchers may be concerned that they will not be able to provide adolescent participants with directed prevention counselling that they are expected to provide (cf. United Nations Programme on HIV/AIDS [UNAIDS] and World Health Organization [WHO], 2012) because adolescents will understandably not make full disclosures of their risks when such risks relate to criminal activity.

This situation holds true for South Africa, where we have outlined several complexities which result from mandatory reporting obligations in the Sexual Offences Act, which require persons who know about a sexual offence against a child to report this to the police. (Bhamjee et al., 2016; Strode and Slack, 2009, 2013; Strode et al., 2013; Strode et al., 2014; Slack et al., 2007a; 2007b; Parliament of South Africa, 2007). If strictly applied, several harms may accrue to adolescent participants.

Adolescent participants might divulge other behaviours that are criminal offences, such as same-sex behaviour. Researching illegal behaviour 
Table 5.3 Ages of consent to sex in selected ESA countries

\begin{tabular}{ll}
\hline Country & Sex \\
\hline Botswana & 16 \\
DRC & 14 (females); 18 (males) \\
Ethiopia & 18 \\
Kenya & 18 \\
Lesotho & 16 \\
Malawi & 13 (females); 12 (males) \\
Mozambique & 18 (females); age for males unclear \\
Namibia & 16 \\
Rwanda & 18 \\
South Africa & 16 \\
Eswatini & 16 \\
Tanzania & 18 \\
Uganda & 18 \\
Zambia & 16 \\
Zimbabwe & 16
\end{tabular}

Source: Grant and Patel, 2016.

Table 5.4 Same-sex behaviour in selected ESA countries

\begin{tabular}{lc}
\hline Country & Illegal? \\
\hline Botswana & No \\
Ethiopia & Yes \\
Kenya & Yes \\
Lesotho & Yes \\
Malawi & Yes \\
Namibia & Yes \\
Eswatini & Yes \\
Uganda & Yes \\
Zambia & Yes \\
Zimbabwe & Yes \\
\hline
\end{tabular}

Source: Grant and Patel, 2016; Motshidiemang, L. v Attorney General and Lesbian, Gays and Bisexuals of Botswana 2019.

is not unethical per se; however, where such behaviours triggers mandatory reporting to authorities, this poses a complex ethical-legal dilemma. Table 5.4 shows that same-sex sexual behaviour is an offence in several countries in ESA. In such settings, potential adolescent participants might be deterred from enrolling in research that identifies such "criminal" behaviour. Researchers may be anxious that if they do not comply with mandatory reporting obligations they might attract criminal sanctions for themselves, but if they do report, then considerable sanctions might accrue to their participants. Here the interests of adolescent participants are pitted against legal obligations (Bhamjee et al., 2016). Researchers and RECs in 
such settings may not have access to national ethics guidelines that provide thoughtful responses to mandatory reporting dilemmas. RECs themselves may be concerned about their own liability if they approve research with an ethically grounded approach that is inconsistent with the law (Strode et al., 2018).

\section{Billboard 3 - stakeholders can advance adolescent HIV prevention research}

The success of clinical trials of HIV prevention products requires cooperation between multiple stakeholders with various expertise and interests (United Nations Programme on HIV/AIDS [UNAIDS] and AIDS Vaccine Advocacy Coalition [AVAC], 2011), and nowhere is this more obvious than in adolescent trials. Next we set out the roles that should be played by a wide range of stakeholders in order to promote the rights of adolescents to benefit from the ever-evolving science of HIV prevention.

\section{Ethics guideline developers}

Guideline developers should ensure that national ethics guidelines encourage the inclusion of adolescents in critical research. More specifically, ethics guidelines should explicitly address the conditions under which waivers of parental consent will be allowed. For example, the Kenyan national guidelines (National AIDS and STI Control Program [NASCOP], 2015) allow this when parental consent is not a "reasonable requirement" or where it will be "inappropriate," such as in studies involving gay or transgender persons (p. 24). An international ethics document from WHO (2018) refers to conditions such as study sensitivity (e.g. sexual activity) and appropriate risk thresholds. Also, national ethics guidelines should address the issue of adolescent privacy; for example, the Kenyan national guidelines (NASCOP, 2015 , p. xiii) argue that privacy for "some components" (e.g. family planning) must be maintained even when a parent gives permission for enrolment. Furthermore, national guidelines must address ethical approaches to mandatory reporting of underage sex and other illegal behaviour. For example, Kenyan guidelines assert that adolescents should understand the "possibility of such [mandatory] reporting" (NASCOP, 2015, p. xiii). In our view, guidelines should explicitly recommend an ethical approach that mitigates against research-related negative social impacts presented by mandatory reporting and its attendant criminal sanctions. For example, the South African Department of Health (2015) national ethics guidelines call for researchers to avoid "thoughtless reporting" (p. 35) to authorities. Guideline developers should ensure that there is a balance between child participation (United Nations General Assembly, 1989), child protection (United Nations General Assembly, 1989) and research facilitation (Strode, 2015). 


\section{RECs or IRBs}

RECs may need to review and approve alternate consent approaches, where parental consent will be a significant impediment to the enrolment of highrisk adolescents (Wallace et al., 2018). Where parental waivers are allowed in national ethics guidance but not in law, then RECs must prepare for a complex ethical-legal dilemma, and we recommend they should approve consent strategies in line with national ethics norms (Strode et al., 2018). We argue that this is appropriate as their primary function is to act ethically and to protect research participants from harm. Nevertheless, they must accept responsibility for this approach and be able to justify their deviation from the law in line with national ethics guidance (Strode et al., 2018). In addition, RECs should fully engage with and review ethical approaches to mandatory reporting. In prior papers we have laid out various components of an ethical approach to South African reporting requirements (Bhamjee et al., 2016; Slack et al., 2007b; Strode et al., 2013; Strode and Slack, 2009, 2013). REC members should vigorously debate the nature and extent of reporting obligations within the context of each HIV prevention study to establish whether reporting will protect adolescents or simply trigger criminal sanctions and further alienation from support. RECs should consider whether there are sufficient protections outlined in the protocol for adolescents engaged in risk behaviour (e.g. through onsite counselling and referral) given the REC's central role in promoting the rights and welfare of participants (Amdur and Bankert, 2011). RECs should carefully balance their core mandate with societal needs to respond to "criminal" behaviour.

In general, RECs should have members that are fluent in laws affecting children and the limitations of such laws, as well as the likely negative impacts on enrolment in socially valuable, otherwise ethical research. RECs should have members prepared to debate and explicitly adopt approaches to adolescent enrolment that are thoughtful, well grounded in relevant ethics principles, and transparently accessible by researchers. REC members should be guided by their primary role to protect research participants rather than adopt the role of "law enforcement." RECs may well find themselves in an ethical-legal conflict regarding consent and reporting approaches (Strode and Slack, 2015; Strode et al., 2018), and in such instances they should seek reassurance about their collective liability if they approve approaches that are inconsistent with the law but are ethical (Strode et al., 2018). The institutions that host RECs should have insurance that protects REC members in the event of a delictual claim for damages resulting from an REC decision (Strode et al., 2018).

\section{Researchers}

As discussed earlier, adolescents may reluctant for their parents to know about sexual activity or orientation (Hosek et al., 2016; Pettifor et al., 2018), 
therefore parental consent may present a considerable barrier to the enrolment of high-risk youth or sexual or gender minority youth (Gilbert et al., 2015; Knopf et al., 2017; WHO, 2018). This concern will exist even where the interviews are conducted in a private space, as it relates to parental knowledge of the nature of the study and its enrolment criteria.

In such instances researchers should explore whether the ethical framework (i.e. national ethics guidelines) will support a waiver of parental consent, even where local laws mandate parental consent, thus allowing self-consent by older adolescents (Strode et al., 2018). For example, South African and Kenyan national guidelines allow such waivers under some instances (SA DOH, 2015; NASCOP, 2015). In South Africa, the REC requires evidence of engagement with community stakeholders, which is also recommended by WHO (2018). In addition, researchers should engage with the responsible REC regarding the optimal consent approach. Where the availability of parents/guardians is an impediment, then seeking to secure permission from an alternative proxy consenter such as a caregiver may be appropriate (Strode and Slack, 2009). However, it is not clear whether adolescents would perceive the involvement of caregivers as a similar deterrent.

With parental waivers and self-consent strategies, researchers must consider "decisional supports" (Knopf et al., 2017) to provide adolescents with additional protections in their decision-making (Gilbert et al., 2015). Adolescents who self-consented to a PrEP trial reported feeling well informed and volunteering freely, yet some wished for additional support at enrolment, which supports the inclusion of such supports (Knopf et al., 2017). This means that researchers should enable adolescents to discuss their decisions with trusted adults (Shah et al., 2018), even chosen by adolescents (Santelli et al., 2017). Researchers should assess capacity for self-consent and carefully assess comprehension of study concepts (Santelli et al., 2003). They should tailor consent processes to target deficiencies in decision-making, such as susceptibility to peer pressure and favouring of short-term rewards over long-term risks (Shah et al., 2018).

Where parental involvement in enrolment will be the consent approach, a privacy strategy must be delineated, that is, which information parents will be informed about. It is clear that children, like adults, have a right to privacy (United Nations General Assembly, 1989), but adult expectations about access to private information must be fully clarified. It follows that some parents may refuse permission for their child's enrolment if the privacy strategy means parents may not be directly informed for components to which adolescents self-consent, as set out elsewhere (Strode and Essack, 2017; Bhamjee et al., 2016; Strode and Slack, 2011, 2013; Strode et al., 2010; Slack et al., 2007a). Careful development of a privacy strategy may well facilitate adolescent enrolment. Also, researchers need to plan an ethical approach to mandatory reporting, namely, an approach that is engages fully with the law that criminalizes the behaviour and any attendant 
requirements to report this to authorities; in other words, the approach should not merely ignore such requirements or demonstrate ignorance of them (Bhamjee et al., 2016). The approach should carefully justify not reporting such consensual behaviour to authorities by invoking ethical principles to promote the welfare of enrolled participants and to avoid research-related harms to them, and the approach should ensure that adolescents engaged in at-risk behaviour receive appropriate help and services for at-risk behaviour (Bhamjee et al., 2016). Researchers should set this out in an application to RECs for a waiver of reporting obligations, as set out elsewhere (Bhamjee et al., 2016).

\section{Adolescents}

Adolescents should understand the personal implications of approaches to consent, privacy and mandatory reporting to be used in the study. To meet obligations for "community" or stakeholder engagement (UNAIDSAVAC, 2011), adolescent representatives should be engaged by the research team to get their insights about the problem of privacy and mandatory reporting, and possible remedies in the form of sensitive ethically nuanced approaches. That is, adolescents should offer their expertise to the research team regarding possible impediments to enrolment and retention. Here adolescent representatives should be engaged by researchers who are trained to interact with this group and who will carefully document their outreach (UNAIDS-AVAC, 2011). Adolescent representatives should be engaged early, so their input is obtained prior to protocols and protocol approaches being finalized or polished. Researchers should obtain inputs regarding how to make the consent, privacy and reporting strategies more acceptable. The participation of adolescents in the research process is underscored in some ethics guidelines (NASCOP, 2015), and our recommendation logically extends their participation to the design of ethical approaches. This is also consistent with their rights as described in the CRC (1989) and with recommendations from commentators (Pettifor et al., 2018).

\section{Policy-makers}

Knee-jerk compliance with reporting laws may draw adolescents (or their partners) into the criminal justice system in a way that attracts potential physical or social harm to participants (Bhamjee et al., 2016; Strode et al., 2013; Strode and Slack, 2009, 2013). Policy-makers should carefully consider amendments to reporting requirements that might deter adolescents from research and may undermine their rights to benefit from scientific progress through their enrolment in socially valuable research (Bhamjee et al., 2016). Policies are needed which underscore the importance of adolescent involvement in HIV prevention research. 


\section{Advocates or activists}

HIV prevention advocates in such settings are uniquely placed to ask difficult questions about how national HIV prevention research agendas are thoughtfully accommodating adolescents. Where laws are identified that hamper such progress, there is no better-placed group to argue for their amendment. Such groups have the skills and voice to advocate for more HIV prevention research involving adolescents so as to ensure that their rights to science are fulfilled, and for reform to laws that impede such rights.

\section{Conclusions}

Adolescents have rights to benefit from scientific advances, as set out in international documents like the CRC. Enrolling them entails consideration of the complications that can arise for study design and implementation. Our message is that such enrolment is feasible with adequate thought and preparation. Adolescent enrolment is also important enough to justify the considerable efforts that must be made to accommodate them. Various stakeholders should recognize their unique contributions to facilitating such enrolment. We reserve the final word for the importance of RECs in such settings, who are required to evaluate whether adolescent protocols are "ethically acceptable" (CIOMS, 2016, p. 8) while often situated in an imperfect legal framework - which necessitates that RECs make sometimes uncomfortable decisions to approve balanced, nuanced, well-justified ethical approaches.

\section{References}

2013. Teddy Bear Clinic for Abused Children and Another v Minister of Justice and Constitutional Development. ZACC 35.

2019. Motshidiemang, L. v Attorney General and Lesbian, Gays and Bisexuals of Botswana 2019. High Court of Botswana held at Gabarone.

Abdool-Karim, Q. \& Dellar, R. 2014. Inclusion of adolescent girls in HIV prevention research - an imperative for an AIDS-free generation. Journal of the International AIDS Society, 17.

African Commission on Human and Peoples' Rights [ACHPR] 1981. African Charter on Human and Peoples' Rights. Organization of African Unity (OAU).

Amdur, R. \& Bankert, E. 2011. Institutional Review Board Member Handbook. Sudbury, MA: Jones and Bartlett Publishing.

Bekker, L.-G., Gill, K. \& Wallace, M. 2015. Pre-exposure prophylaxis for South African adolescents: What evidence? South African Medical Journal, 105, 907-911.

Bekker, L.-G., Slack, C., Lee, S., Shah, S. \& Kapogiannis, B. 2014. Ethical issues in adolescent HIV research in resource-limited countries. JAIDS Journal of Acquired Immune Deficiency Syndromes, 65, S24-S28.

Bhamjee, S., Essack, Z. \& Strode, A. E. 2016. Amendments to the sexual offences act dealing with consensual underage sex: Implications for doctors and researchers. South African Medical Journal, 106, 256-259. 
Busza, J., Strode, A., Dauya, E. \& Ferrand, R.A. 2016. Falling through the gaps: How should HIV programmes respond to families that persistently deny treatment to children? Journal of the International AIDS Society, 19, 20789.

Council for International Organizations of Medical Sciences [CIOMS] 2016. International Ethical Guidelines for Health-related Research Involving Humans. Geneva: CIOMS.

Gilbert, A.L., Knopf, A.S., Fortenberry, J.D., Hosek, S.G., Kapogiannis, B.G. \& Zimet, G. D. 2015. Adolescent self-consent for biomedical human immunodeficiency virus prevention research. Journal of Adolescent Health, 57, 113-119.

Grant, C. \& Patel, P. 2016. UNDP Age of Consent Research Inception Report. Unpublished.

Hosek, S. G., Celum, C., Wilson, C. M., Kapogiannis, B., Delany-Moretlwe, S. \& Bekker, L. G. 2016. Preventing HIV among adolescents with oral PrEP: Observations and challenges in the United States and South Africa. Journal of the International AIDS Society, 19, 21107.

Hosek, S. G. \& Zimet, G. D. 2010. Behavioral considerations for engaging youth in HIV clinical research. JAIDS Journal of Acquired Immune Deficiency Syndromes, 54, S25-S30.

Ivan-Smith, E. \& Johnson, V. 1998. The way forward. In: Johnson, V., Ivan-Smith, E., Gordon, G., Scott-Villiers, P. \& Pridmore, P. (eds.) Stepping Forward: Children and Young People's Participation in the Development Process. Intermediate London: Technology Publications.

Kapogiannis, B. G., Handelsman, E., Ruiz, M. S. \& Lee, S. 2010. Introduction: Paving the way for biomedical HIV prevention interventions in youth. JAIDS Journal of Acquired Immune Deficiency Syndromes, 54, S1-S4.

Knopf, A. S., Ott, M.A., Liu, N., Kapogiannis, B. G., Zimet, G. D., Fortenberry, J. D. \& Hosek, S. G. 2017. Minors' and young adults' experiences of the research consent process in a phase II safety study of pre-exposure prophylaxis for HIV. Journal of Adolescent Health, 61, 747-754.

Macqueen, K. M. \& Abdool-Karim, Q. 2007. Practice brief: Adolescents and HIV clinical trials: Ethics, culture, and context. Journal of the Association of Nurses in AIDS Care, 18, 78-82.

National AIDS and STI Control Programme (NASCOP) \& Kenya Medical Research Institute (KEMRI). 2015. Guidelines for Conducting Adolescent HIV Sexual and Reproductive Health Research in Kenya. Kenya: Government of Kenya.

Nelson, R. M., Lewis, L.L., Struble, K. \& Wood, S.F. 2010. Ethical and regulatory considerations for the inclusion of adolescents in HIV biomedical prevention research. JAIDS Journal of Acquired Immune Deficiency Syndromes, 54, S18-S24.

Nuffield Council on Bioethics. 2015. Children and Clinical Research: Ethical Issues. London: Nuffield Council on Bioethics.

Osmanov, S. 2007. Executive summary and recommendations from WHO/UNAIDS and AAVP consultation on: 'The inclusion of adolescents in HIV vaccine trials', 16-18 March 2006 in Gaborone, Botswana. AIDS (London, England), 21, W1-W10.

Parliament of South Africa. 2007. Criminal Law (Sexual Offences and Related Matters) Amendment Act.

Pettifor, A., Stoner, M., Pike, C. \& Bekker, L.-G. 2018. Adolescent lives matter: Preventing HIV in adolescents. Current Opinion in HIV and AIDS, 13, 265.

Santelli, J. S., Haerizadeh, S. \& McGovern, T. 2017. Inclusion with Protection: Obtaining Informed Consent When Conducting Research with Adolescents. Innocenti Research Briefs no. 2017-05. Florence: UNICEF Office of Research.

Santelli, J. S., Rogers, A. S., Rosenfeld, W. D., Durant, R. H., Dubler, N., Morreale, M., English, A., Lyss, S., Wimberly, Y. \& Schissel, A. 2003. Guidelines for adolescent 
health research: A position paper of the society for adolescent medicine. Journal of Adolescent Health, 33, 396-409.

Scanlon, M.L., Macnaughton, G. \& Sprague, C. 2017. Neglected population, neglected right: Children living with HIV and the right to science. Health and Human Rights, 19, 169.

Shah, S. K., Allison, S. M., Kapogiannis, B. G., Black, R., Dawson, L. \& Erbelding, E. 2018. Advancing Independent Adolescent Consent for Participation in HIV Prevention Research. Journal of Medical Ethics, 44, 431-433.

Slack, C., Strode, A., Fleischer, T., Gray, G. \& Ranchod, C. 2007a. Enrolling adolescents in HIV vaccine trials: Reflections on legal complexities from South Africa. BMC Medical Ethics, 8, 5.

Slack, C., Strode, A. \& Mamashela, M. 2007b. Ethical-legal challenges in adolescent HIV vaccine trials: Research and the law. Southern African Journal of HIV Medicine, 2007, 12-13.

South Africa Department of Health [SA DOH] 2015. Ethics in Health Research: Principles, Processes and Structures. Pretoria: Department of Health.

Strode, A. 2015. A critical review of the regulation of research involving children in South Africa: From self-regulation to hyper-regulation. Journal of South African Law/Tydskrif vir die Suid-Afrikaanse Reg, 2015, 334-346.

Strode, A. \& Essack, Z. 2017. Facilitating access to adolescent sexual and reproductive health services through legislative reform: Lessons from the South African experience. South African Medical Journal, 107, 741-744.

Strode, A., Richter, M., Wallace, M., Toohey, J. \& Technau, K. 2014. Failing the vulnerable: Three new consent norms that will undermine health research with children. Southern African Journal of HIV Medicine, 15, 46-49.

Strode, A., Singh, P., Slack, C. \& Wassenaar, D. 2018. Research ethics committees in a tight spot: Approving consent strategies for child research that are prima facie illegal but are ethical in terms of national guidelines. South African Medical Journal, $108,828-832$.

Strode, A. \& Slack, C. 2009. Sex, lies and disclosures: Researchers and the reporting of under-age sex: Opinion. Southern African Journal of HIV Medicine, 10, 8-10.

Strode, A. \& Slack, C. 2011. Using the concept of 'parental responsibilities and rights' to identify adults able to provide proxy consent to child research in South Africa. South African Journal of Bioethics and Law, 4, 69-73.

Strode, A. \& Slack, C. 2013. Child privacy rights: A 'Cinderella' issue in HIVprevention research. Southern African Journal of HIV Medicine, 14, 108-110.

Strode, A. \& Slack, C. 2015. Child research in South Africa: How do the new regulations help? South African Medical Journal, 105, 899-900.

Strode, A., Slack, C. \& Essack, Z. 2010. Child consent in South African law: Implications for researchers, service providers and policy-makers. South African Medical Journal, 100.

Strode, A., Toohey, J., Slack, C. \& Bhamjee, S. 2013. Reporting underage consensual sex after the Teddy Bear case: A different perspective. South African Journal of Bioethics and Law, 6, 45-47.

S V M 2007. ZACC 18.

UN Committee on the Rights of the Child [CRC] 2003. General comment no. 5 (2003): General measure of implementation of the Convention on the Rights of the Child. 
UNICEF. 2013. Ethical Research Involving Children. Florence: UNICEF Office of Research - Innocenti.

United Nations General Assembly. 1989. Convention on the rights of the child: Adopted and opened for signature, ratification and accession by General Assembly Resolution 44/25 of 20 November 1989.

United Nations Office of the High Commissioner for Human Rights [OHCHR]. 1966. International covenant on economic, social and cultural rights: Adopted and opened for signature, ratification and accession by General Assembly resolution 2200A (XXI) of 16 December 1966, entry into force 3 January 1976, in accordance with article 27.

United Nations Programme on HIV/AIDS [UNAIDS] \& AIDS Vaccine Advocacy Coalition [AVAC]. 2011. Good Participatory Practice: Guidelines for Biomedical HIV Prevention Trials. Geneva: UNAIDS.

United Nations Programme on HIV/AIDS [UNAIDS] \& World Health Organization [WHO]. 2012. Ethical Considerations in Biomedical HIV Prevention Trials [Additional Guidance Point Added in 2012]. Geneva: UNAIDS.

Wallace, M., Middelkoop, K., Smith, P., Pike, C., Bennie, T., Chandia, J., Churchyard, G., Gray, G., Latka, M. \& Mathebula, M. 2018. Feasibility and acceptability of conducting HIV vaccine trials in adolescents in South Africa: Going beyond willingness to participate towards implementation. South African Medical Journal, 108, 291-298.

Wilson, C. M., Wright, P.F., Safrit, J. T. \& Rudy, B. 2010. Epidemiology of HIV infection and risk in adolescents and youth. Journal of Acquired Immune Deficiency Syndromes (1999), 54, S5.

World Health Organization [WHO]. 2018. Guidance on Ethical Considerations in Planning and Reviewing Research Studies on Sexual and Reproductive Health in Adolescents. Geneva: World Health Organization. 